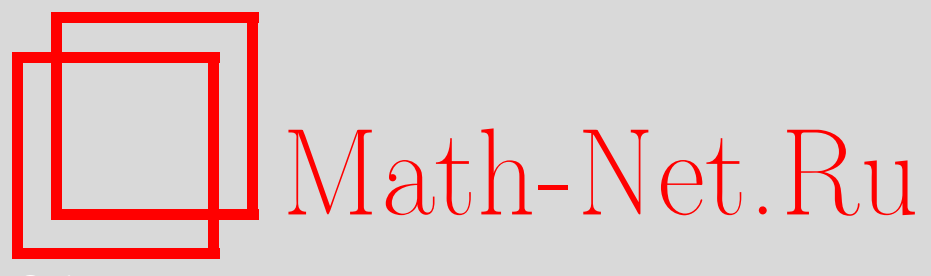

М. А. Мествиришвили, К. А. Модестов, Ю. В. Чугреев, Скалярное поле квинтэссенции в релятивистской теории гравитации, ТМФ, 2007, том 152, номер 3, 551-560

DOI: https://doi.org/10.4213/tmf6110

Использование Общероссийского математического портала Math-Net.Ru подразумевает, что вы прочитали и согласны с пользовательским соглашением http://www . mathnet.ru/rus/agreement

Параметры загрузки:

IP : 3.80 .253 .173

26 апреля 2023 г., 10:33:17

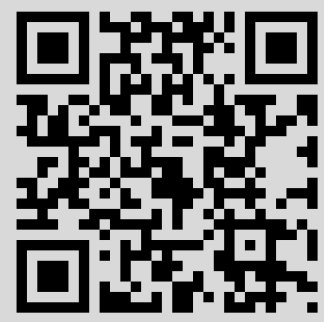




\section{СКАЛЯРНОЕ ПОЛЕ КВИНТЭССЕНЦИИ В РЕЛЯТИВИСТСКОЙ ТЕОРИИ ГРАВИТАЦИИ}

Предлагается вариант теории квинтэссенции, который приводит к ускоренному расширению фридмановской Вселенной в релятивистской теории гравитации. Вещество квинтэссенции создается скалярным полем темной энергии. Показано, что факторизующая лагранжиан скалярного поля $\Phi$ функция $V(\Phi)$ не влияет на развитие Вселенной. Получены соотношения, позволяющие найти явную зависимость $\Phi$ от времени, если только выбрана функция $V(\Phi)$.

Ключевые слова: релятивистская теория гравитации, фридмановская вселенная, квинтэссенция, скалярное поле.

\section{1. ВВЕДЕНИЕ}

На рубеже XXI в. было сделано выдающееся открытие [1] - оказалось, что расширение Вселенной в настоящее время происходит с ускорением. Для объяснения этого явления достаточно предположить, что в однородной и изотропной Вселенной (Вселенной Фридмана) присутствует вещество с необычным уравнением состояния

$$
P_{\mathrm{Q}}=-(1-\nu) \rho_{\mathrm{Q}}
$$

где $P_{\mathrm{Q}}$ - изотропное давление, $\rho_{\mathrm{Q}}$ - плотность массы, а для параметра $\nu$ из общих положений релятивистской теории гравитации (РТГ) [2] следует ограничение вида [3], [4]

$$
0<\nu<\frac{2}{3}
$$

Вещество с таким уравнением состояния было названо "квинтэссенцией".

В данной работе мы предполагаем, что вещество "квинтэссенции" создается скалярным полем. Неудивительно, что моделирование идеальной жидкости с отрицательным давлением обеспечивается довольно необычным лагранжианом скалярного поля.

\footnotetext{
* Институт физики высоких энергий, Протвино, Московская обл., Россия

${ }^{\dagger}$ Московский государственный университет, Москва, Россия. E-mail: ikt@polikvart.ru
} 
Обозначим скалярное поле квинтэссенции как $\Phi\left(x^{\alpha}\right)$ и постулируем, что его плотность лагранжиана имеет вид

$$
L=-\sqrt{-g} V(\Phi)\left(I^{2}\right)^{q},
$$

где $g=\operatorname{det} g_{\mu \nu}, g_{\mu \nu}$ - метрический тензор эффективного риманова пространства [2], $q$ - число, $V(\Phi)>0$ - некоторая функция от $\Phi$ и

$$
I=g^{\alpha \beta} \partial_{\alpha} \Phi \partial_{\beta} \Phi \equiv g^{\alpha \beta} \nabla_{\alpha} \Phi \nabla_{\beta} \Phi .
$$

Здесь $\nabla_{\alpha}-$ ковариантная производная относительно метрики $g_{\alpha \beta}$.

Плотность лагранжиана вида

$$
L=-\sqrt{-g} V(\Phi) F(I),
$$

где $F(I)$ - произвольная функция от $I$, рассматривалась и раньше [5] $]^{1)}$. Наш выбор $F(I)=\left(I^{2}\right)^{q}$ продиктован стремлением получить из полевой теории квинтэссенции уравнение состояния (1.1), которое в рамках РТГ [3], [4] позволяет объяснить ускоренное расширение фридмановской Вселенной.

\section{2. УРАВНЕНИЯ ДВИЖЕНИЯ ДЛЯ ПОЛЯ $\Phi\left(x^{\alpha}\right)$}

Согласно вариационному принципу уравнение движения Эйлера-Лагранжа для поля $\Phi$ есть

$$
\frac{\partial L}{\partial \Phi}-\partial_{\mu} \frac{\partial L}{\partial\left(\partial_{\mu} \Phi\right)}=0 .
$$

После подстановки плотности лагранжиана (1.3) в (2.1) получим

$$
\begin{gathered}
(4 q-1) \frac{d \ln V(\Phi)}{d \Phi} I^{2}-8 q(2 q-1) g^{\alpha \mu} \partial_{\alpha} \Phi\left(\Gamma_{\mu \tau}^{\lambda} g^{\tau \beta} \partial_{\lambda} \partial_{\beta} \Phi-g^{\lambda \beta} \partial_{\beta} \Phi \partial_{\lambda} \partial_{\mu} \Phi\right)- \\
-4 q\left(g^{\mu \tau} \Gamma_{\mu \tau}^{\alpha} \partial_{\alpha} \Phi-g^{\lambda \mu} \partial_{\lambda} \partial_{\mu} \Phi\right) I=0 .
\end{gathered}
$$

Здесь

$$
\Gamma_{\mu \tau}^{\alpha}=\frac{1}{2} g^{\alpha \beta}\left(\partial_{\mu} g_{\beta \tau}+\partial_{\tau} g_{\beta \mu}-\partial_{\beta} g_{\mu \tau}\right)
$$

В терминах ковариантных производных $\nabla_{\alpha}$ уравнение $(2.2)$ запишется в форме

$$
(4 q-1) \frac{d \ln V(\Phi)}{d \Phi} I^{2}+8 q(2 q-1) g^{\alpha \mu} g^{\lambda \beta} \nabla_{\alpha} \Phi \nabla_{\beta} \Phi \nabla_{\lambda} \nabla_{\mu} \Phi+4 q g^{\lambda \mu} \nabla_{\lambda} \nabla_{\mu} \Phi I=0 .
$$

Из плотности лагранжиана (1.3) можно найти симметричную плотность тензора энергии-импульса для поля $\Phi\left(x^{\alpha}\right)$. Согласно Гильберту

$$
T_{\mu \nu}=2 \frac{\delta L}{\delta g^{\mu \nu}} .
$$

Так как $L$ не зависит от производных метрического тензора $g_{\alpha \beta}$, получим

$$
T_{\mu \nu}=2 \frac{\partial L}{\partial g^{\mu \nu}}=\sqrt{-g} V(\Phi)\left(I^{2}\right)^{q-1}\left[g_{\mu \nu} I^{2}-4 q I \partial_{\mu} \Phi \partial_{\nu} \Phi\right] .
$$

\footnotetext{
1) Ссылки на более ранние работы по этой тематике можно найти в работах [5], где для поля $\Phi\left(x^{\alpha}\right)$ с лагранжианом (1.5) употребляется термин “к-эссенция". Мы же сохраним термин "полевая теория квинтэссенции" для любой полевой теории, приводящей к (1.1), поскольку термин “квинтэссенция" по своему происхождению был связан именно с уравнением состояния (1.1).
} 


\section{3. УРАВНЕНИЕ ДВИЖЕНИЯ ДЛЯ ПОЛЯ $\Phi$ И ЕГО ТЕНЗОР ЭНЕРГИИ-ИМПУЛЬСА В ОДНОРОДНОЙ И ИЗОТРОПНОЙ ВСЕЛЕННОЙ}

Для однородной и изотропной Вселенной метрические тензоры $g_{\alpha \beta}$ и $g^{\alpha \beta}$ имеют вид [2]

$$
\begin{aligned}
& g_{00}=1 ; \quad g_{11}=g_{22}=g_{33}=-a_{\max }^{4} a^{2} ; \quad g_{\alpha \beta}=0, \quad \alpha \neq \beta, \\
& g^{00}=1 ; \quad g^{11}=g^{22}=g^{33}=-\frac{1}{a_{\max }^{4} a^{2}} ; \quad g^{\alpha \beta}=0, \quad \alpha \neq \beta .
\end{aligned}
$$

Здесь $a(\tau)$ - масштабный фактор и $a_{\max }<\infty$ - его максимальное значение. Величина $\sqrt{-g}$ равна

$$
\sqrt{-g}=a_{\max }^{6} a^{3} .
$$

Для того чтобы поле $\Phi\left(x^{\alpha}\right)$ не нарушало однородности и изотропии Вселенной, необходимо предположить, что $\Phi\left(x^{\alpha}\right)$ зависит только от $\tau$. Тогда согласно (1.4) и $(3.1 б)$

$$
I=\left(\partial_{0} \Phi\right)^{2}
$$

и для $T_{\mu \nu}$ из формулы $(2.4)$ с учетом (3.1) получим выражения

$$
\begin{gathered}
T_{00}=(1-4 q) a_{\max }^{6} a^{3} V(\Phi)\left[\left(\partial_{0} \Phi\right)^{2}\right]^{2 q} ; \\
T_{11}=T_{22}=T_{33}=-a_{\max }^{10} a^{5} V(\Phi)\left[\left(\partial_{0} \Phi\right)^{2}\right]^{2 q} ; \quad T_{\mu \nu}=0, \quad \mu \neq \nu .
\end{gathered}
$$

Обычно предполагается, что во фридмановской Вселенной вещество описывается плотностью тензора энергии-импульса идеальной жидкости:

$$
T_{\mu \nu}=\sqrt{-g}\left[(\rho+p) U_{\mu} U_{\nu}-g_{\mu \nu} p\right] .
$$

Так как для однородной и изотропной Вселенной 4-скорость $U_{\mu}$ имеет вид

$$
U_{0}=1, \quad U_{k}=0,
$$

из выражения (3.5) с учетом (3.1) находим

$$
\begin{gathered}
T_{00}=a_{\max }^{6} a^{3} \rho, \\
T_{11}=T_{22}=T_{33}=a_{\max }^{10} a^{5} p ; \quad T_{\mu \nu}=0, \quad \mu \neq \nu .
\end{gathered}
$$

Распространяя гипотезу о представлении плотности тензора энергии-импульса поля вещества в виде плотности энергии-импульса идеальной жидкости на квинтэссенцию, после сравнения (3.3) и (3.4) с (3.6) и (3.7), соответственно, получим

$$
\begin{aligned}
& \rho_{Q}=(1-4 q) V(\Phi)\left[\left(\partial_{0} \Phi\right)^{2}\right]^{2 q}, \\
& P_{Q}=-V(\Phi)\left[\left(\partial_{0} \Phi\right)^{2}\right]^{2 q} .
\end{aligned}
$$

Следовательно,

$$
P_{Q}=-\frac{1}{(1-4 q)} \rho_{Q}
$$


Полагая

$$
1-4 q=\frac{1}{1-\nu}
$$

мы видим, что выражение (3.10) переходит в (1.1). Это дает основание считать поле $\Phi$ с плотностью лагранжиана (1.3) полем квинтэссенции, если только

$$
q=-\frac{\nu}{4(1-\nu)}
$$

Учитывая ограничение (1.2) для параметра $\nu$, из (3.12) находим

$$
-\frac{1}{2}<q<0
$$

Таким образом, плотность лагранжиана для квинтэссенции примет вид

$$
L=-\sqrt{-g} V(\Phi)\left(I^{2}\right)^{\nu /(4(1-\nu))},
$$

а плотность и давление для этого поля в однородной и изотропной Вселенной будут равны

$$
\begin{aligned}
\rho_{Q} & =\frac{1}{1-\nu} V(\Phi)\left[\left(\partial_{0} \Phi\right)^{2}\right]^{\nu /(2(1-\nu))}, \\
P_{Q} & =-V(\Phi)\left[\left(\partial_{0} \Phi\right)^{2}\right]^{\nu /(2(1-\nu))} .
\end{aligned}
$$

Чтобы найти явный вид (3.15) и (3.16), необходимо решить уравнение движения (2.2). Так как для метрики (3.1a), (3.1б) коэффициенты связности имеют вид

$$
\Gamma_{00}^{0}=0, \quad \Gamma_{i k}^{0}=a_{\max }^{4} a \partial_{0} a \delta_{i k} ; \quad g^{\mu \tau} \Gamma_{\mu \tau}^{0}=-\frac{3}{a} \partial_{0} a,
$$

то из (2.2) с учетом (3.12) найдем

$$
-\frac{1}{1-\nu} \frac{d \ln V(\Phi)}{d \Phi}\left(\partial_{0} \Phi\right)^{4}-\frac{3 \nu}{(1-\nu)} \frac{1}{a} \partial_{0} a\left(\partial_{0} \Phi\right)^{3}+\frac{\nu}{(1-\nu)^{2}}\left(\partial_{0} \Phi\right)^{2} \partial_{0}^{2} \Phi=0 .
$$

Поделив это выражение на $-\left(\partial_{0} \Phi\right)^{3} /(1-\nu)^{2}$ и принимая во внимание тождество

$$
\frac{d \ln V(\Phi)}{d \Phi} \partial_{0} \Phi \equiv \partial_{0} \ln V(\Phi)
$$

получим

$$
\partial_{0} \ln \left[(V(\Phi))^{1-\nu}\left|\partial_{0} \Phi\right|^{-\nu} a^{3 \nu(1-\nu)}\right]=0
$$

т.e.

$$
V(\Phi)\left|\partial_{0} \Phi\right|^{\nu /(1-\nu)}=\frac{\Lambda}{a^{3 \nu}}, \quad \Lambda=\text { const }>0 .
$$

Подставляя это выражение в (3.15) и (3.16), окончательно находим

$$
\begin{aligned}
& \rho_{\mathrm{Q}}=\frac{\Lambda}{1-\nu} \frac{1}{a^{3 \nu}}, \\
& P_{\mathrm{Q}}=-\frac{\Lambda}{a^{3 \nu}} .
\end{aligned}
$$


Хорошо известно [2], [4], что для уравнения состояния (1.1) ковариантный закон сохранения $\nabla_{\mu} T^{\mu \nu}=0$, где $T^{\mu \nu}$ задается соотношением (3.5), во фридмановской Вселенной приводит к формулам

$$
\rho_{Q}=\frac{B}{a^{3 \nu}}, \quad P_{Q}=-(1-\nu) \frac{B}{a^{3 \nu}},
$$

где $B=$ const $>0$.

После переопределения постоянной $\Lambda$

$$
\Lambda=(1-\nu) B
$$

мы видим, что выражения (3.18), (3.19) переходят в (3.20), что означает эквивалентность уравнения движения (2.2) и ковариантного закона сохранения $\nabla_{\mu} T^{\mu \nu}=0$, дополненного соотношением (1.1).

Важно отметить, что для получения основных формул (3.18) и (3.19), определяющих эволюцию однородной и изотропной Вселенной, не требуется конкретизации функции $V(\Phi)$.

Обозначим через $a_{\min }$ и $\Phi(0)$ значения масштабного фактора $a(\tau)$ и поля $\Phi(\tau)$ в момент начала цикла расширения Вселенной $\tau=0$. Тогда формулу (3.17) можно переписать в виде

$$
\int_{\Phi(0)}^{\Phi(\tau)} \frac{d \Phi}{V(\Phi)^{(1-\nu) / \nu}}=\Lambda^{(1-\nu) / \nu} \int_{0}^{\tau} a^{3(1-\nu)} d \tau .
$$

Фридмановская Вселенная в РТГ развивается во времени циклически - ее масштабный фактор $a(\tau)$ меняется в области

$$
0<a_{\min } \leqslant a(\tau) \leqslant a_{\max }<\infty .
$$

Поэтому интеграл

$$
J(\tau)=\int_{0}^{\tau} a^{3(1-\nu)} d \tau
$$

конечен. Следовательно, и левая часть уравнения (3.21) должна быть конечной. Это налагает некоторое, хотя и очень слабое, ограничение на функцию $V(\Phi)$.

Используя известные в РТГ результаты, интеграл (3.23) можно выразить через наблюдаемые параметры, что облегчит дальнейший анализ. С этой целью перепишем (3.23) в виде

$$
J(\tau)=\int_{0}^{a(\tau)} a^{2-3 \nu}\left(a \frac{d \tau}{d a}\right) d a .
$$

Для стадии расширения Вселенной согласно РТГ [2], [4]

$$
a \frac{d \tau}{d a}=\left[\frac{8 \pi G \rho}{3}-\frac{m^{2}}{6}\left(1-\frac{3}{2 \beta^{4} a^{2}}+\frac{1}{2 a^{6}}\right)\right]^{-1 / 2},
$$

где $\beta=a_{\max }, m^{2}=\left(m_{\mathrm{g}} c^{2} / \hbar\right)^{2}, m_{\mathrm{g}}$ - масса гравитона $\left(m_{\mathrm{g}} \cong 3.6 \cdot 10^{-66} \Gamma[4]\right)$, а $\rho-$ полная плотность массы во Вселенной:

$$
\rho=\rho_{\mathrm{r}}+\rho_{\mathrm{m}}+\rho_{\mathrm{Q}}
$$


Здесь $\rho_{\mathrm{r}}$ - плотность массы радиационной фазы, $\rho_{\mathrm{m}}-$ плотность массы барионодоминантной фазы и $\rho_{\mathrm{Q}}-$ плотность массы фазы квинтэссенции.

Введем следующие обозначения:

1) $x=a(\tau) / a_{0}$, где $a_{0}$ - значение масштабного фактора $a(\tau)$ в настоящий момент времени $\tau=\tau_{\text {pres }}$. По порядку величины $a_{0} \approx 5 \cdot 10^{9}[4]$;

2) постоянную Хаббла

$$
H=\left(\frac{1}{a} \frac{d a}{d \tau}\right)_{\tau=\tau_{\text {pres }}}
$$

и связанную с ней критическую плотность $\rho_{\tilde{n}}^{0}$ :

$$
H^{2}=\frac{8 \pi G}{3} \rho_{\tilde{n}}^{0}
$$

3) относительные плотности

$$
\Omega_{\mathrm{r}}^{0}=\frac{\rho_{\mathrm{r}}^{0}}{\rho_{c}^{0}}, \quad \Omega_{\mathrm{m}}^{0}=\frac{\rho_{\mathrm{m}}^{0}}{\rho_{c}^{0}}, \quad \Omega_{\mathrm{Q}}^{0}=\frac{\rho_{\mathrm{Q}}^{0}}{\rho_{c}^{0}},
$$

где $\rho_{\mathrm{r}}^{0}, \rho_{\mathrm{m}}^{0}$ и $\rho_{\mathrm{Q}}^{0}-$ плотности масс радиационной и барионной фаз и фазы квинтэссенции, соответственно, в момент $\tau=\tau_{\text {pres }}$.

Так как [2], [4]

$$
\rho_{\mathrm{r}}=\frac{A_{\mathrm{r}}^{0}}{a^{4}}, \quad \rho_{\mathrm{m}}=\frac{A_{\mathrm{m}}}{a^{3}}, \quad \rho_{\mathrm{Q}}=\frac{A_{\mathrm{Q}}}{a^{3 \nu}},
$$

где $A_{\mathrm{r}}, A_{\mathrm{m}}$ и $A_{\mathrm{Q}}$ - некоторые постоянные, то в терминах переменной $x$ имеем

$$
\rho_{\mathrm{r}}=\frac{\rho_{\mathrm{r}}^{0}}{x^{4}}, \quad \rho_{\mathrm{m}}=\frac{\rho_{\mathrm{m}}^{0}}{x^{3}}, \quad \rho_{\mathrm{Q}}=\frac{\rho_{\mathrm{Q}}^{0}}{x^{3 \nu}},
$$

поэтому

$$
\frac{8 \pi G}{3} \rho=H^{2}\left(\frac{\Omega_{\mathrm{r}}^{0}}{x^{4}}+\frac{\Omega_{\mathrm{m}}^{0}}{x^{3}}+\frac{\Omega_{\mathrm{Q}}^{0}}{x^{3 \nu}}\right) .
$$

С учетом выражений (3.25) и (3.27) для интеграла $J(\tau)$ получим

$$
J(\tau)=\frac{a_{0}^{3(1-\nu)}}{H} \int_{x_{\min }}^{x(\tau)} x^{2-3 \nu}\left[\frac{\Omega_{\mathrm{r}}^{0}}{x^{4}}+\frac{\Omega_{\mathrm{m}}^{0}}{x^{3}}+\frac{\Omega_{\mathrm{Q}}^{0}}{x^{3 \nu}}-\frac{f^{2}}{6}\left(1-\frac{3}{2 \beta^{4} a_{0}^{2} x^{2}}+\frac{1}{2 a_{0}^{6} x^{6}}\right)\right]^{-1 / 2} d x,
$$

где

$$
f=\frac{m}{H}=\frac{m_{\mathrm{g}} c^{2}}{\hbar H}, \quad x_{\min }=\frac{a_{\mathrm{min}}}{a_{0}} .
$$

Выражение (3.28) содержит параметры, значения которых могут быть установлены экспериментально [4]. Например, современные наблюдательные данные приводят к следующим значениям для относительных плотностей [6]:

$$
\Omega_{\mathrm{r}}^{0} \approx 0.6 \cdot 10^{-4}, \quad \Omega_{\mathrm{m}}^{0} \approx 0.3, \quad \Omega_{\mathrm{Q}}^{0} \approx 0.7 .
$$

В радиационно-доминирующей области

$$
a_{\min } \leqslant a(\tau) \leqslant a_{\mathrm{r}},
$$


где $a_{\mathrm{r}}$ - значение масштабного фактора, при котором в квадратном корне под интегралом (3.28) ведущими членами становятся $\Omega_{\mathrm{r}}^{0}$ и $f^{2} /\left(12 a_{0}^{6} x^{6}\right)$, а остальными можно пренебречь; для $J(\tau)$ можно использовать следующее приближенное выражение:

$$
J_{r}(\tau) \cong \frac{a_{0}^{3(1-\nu)}}{H \sqrt{\Omega_{\mathrm{r}}^{0}}} \int_{x_{\min }}^{x(\tau)} \frac{x^{5-3 \nu} d x}{\sqrt{x^{2}-x_{\min }^{2}}} .
$$

Здесь

$$
x_{\min } \equiv \frac{a_{\min }}{a_{0}}=\left(\frac{f^{2}}{12 a_{0}^{6} \Omega_{\mathrm{r}}^{0}}\right)^{1 / 2}
$$

a $x(\tau) \leqslant x_{\mathrm{r}}=\left(a_{r} / a_{0}\right)$.

После интегрирования из выражения (3.31) в радиационно-доминирующей области получим

$$
J(\tau) \cong \frac{a_{\min }^{3(1-\nu)}}{H \sqrt{\Omega_{\mathrm{r}}^{0}}} x_{\min }^{2}\left[\left(\frac{a}{a_{\min }}\right)^{2}-1\right]^{1 / 2} F\left(-2+\frac{3 \nu}{2} ; 1 ; \frac{3}{2} ; 1-\left(\frac{a}{a_{\min }}\right)^{2}\right) .
$$

В малой окрестности $a_{\min }$ отсюда находим

$$
J_{r}(\tau) \cong \frac{a_{\min }^{3(1-\nu)}}{H \sqrt{\Omega_{\mathrm{r}}^{0}}} x_{\min }^{2}\left[\left(\frac{a}{a_{\min }}\right)^{2}-1\right]^{1 / 2} .
$$

Вычислим теперь выражение для $J(\tau)$ на стадии квинтэссенции. Введем обозначение $x_{\mathrm{Q}}=a_{\mathrm{Q}} / a_{0}$ где $a_{Q}$ - значение масштабного фактора, начиная с которого во Вселенной доминирует квинтэссенция.

Из условий

$$
\frac{\Omega_{\mathrm{r}}^{0}}{x^{4}} \ll \frac{\Omega_{\mathrm{Q}}^{0}}{x^{3 \nu}}, \quad \frac{\Omega_{\mathrm{m}}^{0}}{x^{3}} \ll \frac{\Omega_{\mathrm{Q}}^{0}}{x^{3 \nu}}
$$

с учетом (3.30) легко найти оценку

$$
\left(\frac{3}{7}\right)^{1 /(3(1-\nu))}<1 \ll x_{\mathrm{Q}} \leqslant x_{\max } .
$$

Перепишем (3.28) в виде

$$
J(\tau) \approx J_{1}+\frac{a_{0}^{3(1-\nu)} \sqrt{6}}{H f} \int_{x_{\mathrm{Q}}}^{x(\tau)} \frac{x^{2-3 \nu / 2} d x}{\sqrt{\Omega_{\mathrm{Q}}^{0} 6 / f^{2}-x^{3 \nu}}},
$$

где

$$
J_{1}=\frac{a_{0}^{3(1-\nu)}}{H} \int_{x_{\min }}^{x_{\mathrm{Q}}} x^{2-3 \nu}\left[\frac{\Omega_{\mathrm{r}}^{0}}{x^{4}}+\frac{\Omega_{\mathrm{m}}^{0}}{x^{3}}+\frac{\Omega_{\mathrm{Q}}^{0}}{x^{3 \nu}}-\frac{f^{2}}{6}\left(1-\frac{3}{2 \beta^{4} a_{0}^{2} x^{2}}+\frac{1}{2 a_{0}^{6} x^{6}}\right)\right]^{-1 / 2} d x
$$

Входящий в выражение (3.36) интеграл

$$
I=\int_{x_{\mathrm{Q}}}^{x(\tau)} \frac{x^{2-3 \nu / 2} d x}{\sqrt{\Omega_{\mathrm{Q}}^{0} 6 / f^{2}-x^{3 \nu}}}
$$


приближенно равен

$$
I \approx \frac{2}{3(2-\nu)}\left(\frac{a(\tau)}{a_{0}}\right)^{3(1-\nu / 2)} \frac{1}{\sqrt{t_{\max }}}\left[F\left(\frac{1}{2} ; \frac{1}{\nu}-\frac{1}{2} ; \frac{1}{\nu}+\frac{1}{2} ; \frac{a^{3 \nu}}{a_{0}^{3 \nu} t_{\max }}\right)-\left(\frac{a_{\mathrm{Q}}}{a(\tau)}\right)^{3(1-\nu / 2)}\right],
$$

где

$$
t_{\max } \equiv\left(\frac{a_{\max }}{a_{0}}\right)^{3 \nu}=\frac{\Omega_{\mathrm{Q}}^{0} 6}{f^{2}} .
$$

Подставляя (3.38) в (3.36), получим приближенное выражение $J(\tau)$ для стадии квинтэссенции:

$$
\begin{aligned}
J(\tau) \approx J_{1} & +\frac{2}{3(2-\nu) H \sqrt{\Omega_{\mathrm{Q}}^{0}}}\left(\frac{a(\tau)}{a_{0}}\right)^{3(1-\nu / 2)} \times \\
\times & {\left[F\left(\frac{1}{2} ; \frac{1}{\nu}-\frac{1}{2} ; \frac{1}{\nu}+\frac{1}{2} ; \frac{a^{3 \nu}}{a_{0}^{3 \nu} t_{\max }}\right)-\left(\frac{a_{\mathrm{Q}}}{a(\tau)}\right)^{3(1-\nu / 2)}\right] . }
\end{aligned}
$$

Чтобы выразить $J$ в терминах $\tau$, необходимо найти $a$ как функцию $\tau$, т.е. разрешить уравнение (3.25). Следуя работе [4], запишем его в обозначениях, введенных в п. 1-3 после равенства (3.26). Тогда решение можно представить в виде

$$
\tau=\frac{1}{H} \int_{x_{\min }}^{x(\tau)} \frac{1}{x}\left[\frac{\Omega_{\mathrm{r}}^{0}}{x^{4}}+\frac{\Omega_{\mathrm{m}}^{0}}{x^{3}}+\frac{\Omega_{\mathrm{Q}}^{0}}{x^{3 \nu}}-\frac{f^{2}}{6}\left(1-\frac{3}{2 \beta^{4} a_{0}^{2} x^{2}}+\frac{1}{2 a_{0}^{6} x^{6}}\right)\right]^{-1 / 2} d x .
$$

Анализ этого выражения проведен в работе [4], где показано, что в окрестности $\tau \approx 0$

$$
a(\tau) \approx a_{\min }\left(1+\frac{4 \pi G}{3} \rho_{\max } \tau^{2}\right) .
$$

В той области развития Вселенной, где массовыми членами (т.е. пропорциональными $f$ членами под знаком интеграла (3.41)) можно пренебречь, зависимость $a=a(\tau)$ известна, и она такая же, как в общей теории относительности. Поэтому мы ее здесь не приводим.

При больших $a(\tau)$, когда во Вселенной доминирует квинтэссенция, выражение (3.41) можно представить в виде

$$
\tau \approx \tau_{1}+\frac{\sqrt{6}}{H f} \int_{x_{\mathrm{Q}}}^{x(\tau)} \frac{x^{3 \nu / 2-1} d x}{\sqrt{\Omega_{Q}^{0} 6 / f^{2}-x^{3 \nu}}}
$$

где временно́й интервал

$$
\tau_{1}=\frac{1}{H} \int_{x_{\min }}^{x_{\mathrm{Q}}} \frac{1}{x}\left[\frac{\Omega_{\mathrm{r}}^{0}}{x^{4}}+\frac{\Omega_{\mathrm{m}}^{0}}{x^{3}}+\frac{\Omega_{\mathrm{Q}}^{0}}{x^{3 \nu}}-\frac{f}{6}\left(1-\frac{3}{2 \beta^{4} a_{0}^{2} x^{2}}+\frac{1}{2 a_{0}^{6} x^{6}}\right)\right]^{1 / 2} d x
$$

равен времени от начала расширения до момента $\tau_{1}$ - времени наступления преобладания квинтэссенции во Вселенной, причем $\tau_{1} \leqslant \tau_{\text {pres }}$. Так как

$$
\int_{x_{\mathrm{Q}}}^{x(\tau)} \frac{x^{3 \nu / 2-1} d x}{\sqrt{\Omega_{\mathrm{Q}}^{0} 6 / f^{2}-x^{3 \nu}}} \approx \frac{1}{3 \nu} \arccos \left(1-2 \frac{x^{3 \nu}}{t_{\max }}\right)-\frac{2}{3 \nu} \frac{x_{\mathrm{Q}}^{3 \nu}}{\sqrt{t_{\max }}},
$$


то после подстановки этого выражения в (3.43) можно найти

$$
\left(\frac{a(\tau)}{a_{0}}\right)^{3 \nu} \approx \frac{3 \Omega_{\mathrm{Q}}^{0}}{f^{2}}\left[1-\cos \left(\sqrt{\frac{3}{2}} f\left(H \nu\left(\tau-\tau_{1}\right)+\frac{2}{3}\left(\frac{a_{Q}}{a_{0}}\right)^{3 \nu} \frac{1}{\sqrt{\Omega_{\mathrm{Q}}^{0}}}\right)\right)\right]
$$

Так как $x_{\max }^{3 \nu} \equiv\left(a_{\max } / a_{0}\right)^{3 \nu}=6 \Omega_{\mathrm{Q}}^{0} / f^{2}$ и $\tau=\tau_{\max } \gg \tau_{1}$ при $a=a_{\max }$, из (3.45) получаем

$$
\sqrt{\frac{3}{2}} f H \nu \tau_{\max } \approx \pi
$$

откуда можно найти $\tau_{\max }$. $\mathrm{B}$ окрестности $\tau \approx \tau_{\max }$ с помощью (3.45) нетрудно установить, что

$$
a(\tau) \approx a_{\max }\left[1-\frac{\pi^{2}}{4}\left(1-\frac{\tau}{\tau_{\max }}\right)^{2}\right]^{1 / 3 \nu} .
$$

Подставляя (3.42) в (3.34), получим значение $J(\tau)$ в окрестности $\tau \approx 0$ :

$$
J_{\mathrm{r}}(\tau) \approx \frac{a_{\min }^{3(1-\nu)}}{H \sqrt{\Omega_{\mathrm{r}}^{0}}} x_{\min }^{2}\left(\frac{8 \pi G \rho_{\max }}{3}\right)^{1 / 2} \tau .
$$

Согласно теореме о среднем, для $J_{1}$ (см. выражение (3.37)) имеем

$$
J_{1}=\left(x_{0} x_{1}\right)^{3(1-\nu)} \tau_{1},
$$

где $x_{1}$ - некоторое число из интервала $\left(x_{\min }, x_{\mathrm{Q}}\right)$, а $\tau_{1}$ задано формулой $(3.44)$.

Принимая во внимание определение $t_{\max }(3.39)$, а также $(3.47)$, находим

$$
\left(\frac{a}{a_{0}}\right)^{3 \nu} \frac{1}{t_{\max }}=\left(\frac{a}{a_{\max }}\right)^{3 \nu} \approx 1-\frac{\pi^{2}}{4}\left(1-\frac{\tau}{\tau_{\max }}\right)^{2} .
$$

Учитывая $(3.47),(3.49)$ и (3.50) в выражении $(3.40)$, для $J(\tau)$ в окрестности $\tau \approx \tau_{\max }$ получим

$$
\begin{aligned}
J(\tau) \approx( & \left.x_{0} x_{1}\right)^{3(1-\nu)} \tau_{1}+\frac{2}{3(2-\nu) H \sqrt{\Omega_{\mathrm{Q}}^{0}}}\left(\frac{a_{\max }}{a_{0}}\right)^{3(1-\nu / 2)} \times \\
& \times\left[1-\frac{\pi^{2}}{4}\left(1-\frac{\tau}{\tau_{\max }}\right)^{2}\right]^{(2-\nu) / 2 \nu} F\left(\frac{1}{2} ; \frac{1}{\nu}-\frac{1}{2} ; \frac{1}{\nu}+\frac{1}{2} ; 1-\frac{\pi^{2}}{4}\left(1-\frac{\tau}{\tau_{\max }}\right)^{2}\right) .
\end{aligned}
$$

Явное выражение для поля $\Phi(\tau)$ может быть найдено из (3.21), если задать вид функции $V(\Phi)$, но сама фридмановская модель на него ограничений не налагает. Другими словами, различные теории квинтэссенции с различными лагранжианами (1.3), отвечающими различным $V(\Phi)$, приводят к одинаковым значениям для $\rho_{\mathrm{Q}}$ и $P_{\mathrm{Q}}$. Следовательно, все они одинаково влияют на развитие Вселенной во времени.

В ряде работ в качестве $V(\Phi)$ выбирают степенну́ю функцию $V(\Phi) \sim \Phi^{-\alpha}$, где $\alpha-$ некоторое положительное число. Очевидно, что в этом случае из интеграла (3.21) можно найти явное выражение $\Phi(\tau)$ через $J(\tau)$. 
Благодарности. Авторы выражают глубокую благодарность А. А. Логунову за постоянный интерес к работе и многочисленные обсуждения. М. А. Мествиришвили благодарит также сотрудников ОТФ ИФВЭ Ю. М. Зиновьева, В. В. Киселева, А. В. Разумова, А. П. Самохина, В. О. Соловьева и Ю.Г. Строганова за полезные обсуждения.

\section{Список литературы}

[1] A. G. Ries, A. V. Filippenko, P. Challis et al., Astron. J. Suppl. Ser., 116 (1988), 1009-1038; S. Perlmutter, G. Aldering, M. della Valle et al., Nature, 391 (1988), 51-54; S. Perlmutter, G. Aldering, G. Goldhaber et al., Astrophys. J, 517 (1999), 565-586; C. L. Bennett, A. J. Banday, K. M. Gorski et al., Astrophys. J, 64 (1996), L1-L4; S. Hanany, P. Ade, A. Balbi et al., Astrophys. J, 545 (2000), L5-L9; P. Bernardis, P. A. R. Ade, J. J. Bock et al., Nature, 404 (2000), 955-959; A. Benoit, P. Ade, A. Amblard et al., Astron. Astrophys., 399 (2003), L19-L23; L25-L30; C. L. Bennet, M. Halpern, G. Hinshaw et al., Astrophys. J. Suppl. Ser., 148 (2003), 1-27; D. N. Spergel, L. Verde, H. V. Peiris et al., Astrophys. J. Suppl. Ser., 148 (2003), 175-194; W. J. Percival, C. M. Baugh, J. Bland-Hawthorn et al., Mon. Not. R. Astron. Soc., 327 (2001), 1297-1306; L. Verde, A. F. Heavens, W. J. Percival et al., Mon. Not. R. Astron. Soc., 335 (2002), 432-440; L. Verde, H. V. Peiris, D. N. Spergel et al., Astrophys. J. Suppl. Ser., 148 (2003), 195-212; D. G. York, J. Adelman, J. E. Anderson et al., Astron. J, 120 (2000), 1579-1587; C. Stroughton, R. H. Lupton, M. Bernardi et al., Astron. J, 123 (2002), 485-548; K. Abazajian, J. K. Adelman-McCarthy, M. A. Agueros et al., Astron. J, 126 (2000), 2081-2086.

[2] А.А. Логунов, Теория гравитационного поля, Наука, М., 2000.

[3] V. L. Kalashnikov, X-matter induced cosmological scenarios in the relativistic theory of gravity, gr-qc/0109060; Constraints on the cosmological parameters in the relativistic theory of gravitation, gr-qc/0202084.

[4] С. С. Герштейн, А.А. Логунов, М. А. Мествиришвили, Н.П. Ткаченко, ЭЧАЯ, 36:5 (2005), 1003-1050.

[5] L. P. Chimento, A. Feinstein, Mod. Phys. Lett. A, 19 (2004), 761-768; R. J. Scherrer, Phys. Rev. Lett, 93 (2004), 011301; R. Das, T. W. Kephart, R. J. Scherrer, Tracking quintessence and k-essence in a general cosmological background, gr-qc/0609014.

[6] M. Tegmark, M. A. Strauss, M. R. Blanton et al., Phys. Rev. D, 69 (2004), 103501. 